Mypinder S. Sekhon

Donald E. Griesdale

Chiara Robba

Nick McGlashan

Edward Needham

Katherine Walland

Alyssa C. Shook

Peter Smielewski

Marek Czosnyka

Arun K. Gupta

David K. Menon

\section{Erratum to: Optic nerve sheath diameter on computed tomography is correlated with simultaneously measured intracranial pressure in patients with severe traumatic brain injury}

Published online: 18 November 2014

(C) Springer-Verlag Berlin Heidelberg and ESICM 2014

The online version of the original article can be found under doi:10.1007/s00134-014-3392-7.

M. S. Sekhon · D. E. Griesdale

Division of Critical Care Medicine, Department of Medicine, Vancouver General Hospital, University of British Columbia, Room 2438, Jim Pattison Pavilion, 2nd Floor, 855 West 12th Avenue, Vancouver, BC V5Z 1M9, Canada

M. S. Sekhon

e-mail: mypindersekhon@gmail.com

Tel.: 604-875-5949

M. S. Sekhon - C. Robba · N. McGlashan ·

E. Needham · K. Walland · P. Smielewski .

M. Czosnyka · A. K. Gupta · D. K. Menon ( $)$

Neurocritical Care Unit, Addenbrooke's Hospital, Cambridge

University, Cambridge University Hospitals Trust, Hills Road, Box

1, Cambridge CB2 0QQ, UK

e-mail: dkm13@wbic.cam.ac.uk

D. E. Griesdale

Department of Anaesthesiology, Pharmacology and Therapeutics, Vancouver General Hospital, University of British Columbia, West 12th Avenue, Vancouver, BC V5Z 1M9, Canada

\section{E. Griesdale}

Centre for Clinical Epidemiology and Evaluation, Vancouver Coastal Health Research Institute, University of British Columbia, 899 West 12th Avenue, Vancouver, BC V5Z 1M9, Canada

A. C. Shook

The Heart Centre, St. Paul's Hospital, 1181 Burrard Street,

Vancouver, BC V6Z 1Y6, Canada
Erratum to: Intensive Care Med (2014)

\section{0:1267-1274}

DOI 10.1007/s00134-014-3392-7

Owing to an oversight by the authors during editing and revision of the manuscript incorrect information on the process of ethical approval appeared in the original publication. The erroneous text read: "The protocol was approved by the Research Ethics Boards at the University of Cambridge who waived the requirement for written informed consent."

The correct text is as follows:

Consecutive traumatic brain-injured patients with a clinical need for ICP monitoring were included for analysis. Informed consent was obtained from all patients (or their next of kin) for the use of collected data for research purposes. The study was approved by the relevant research ethics committee (29 REC 97/291). 